\title{
Prevalence of Mental Disorders and the Pandemic of COVID-19: A Contemporary Literature Review
}

\author{
Amal Ibrahim Khalil
}

Professor of Psychiatry and Mental Health Nursing, Menoufia University, Egypt

King Saud Bin Abd Al-Aziz University for Health Sciences, College of Nursing, Jeddah, Kingdom of Saudi Arabia

DOI: $\underline{10.36348 / \text { sjbr.2020.v05i09.001 }}$

| Received: 28.08.2020 | Accepted: 05.09.2020 | Published: 13.09.2020

*Corresponding author: Amal Ibrahim Khalil

Abstract

Background: The association between COVID-19 and mental illness started due to the inevitable fear and anxiety about the emergence of a new disease. A future so unpredictable can be overwhelming and can cause strong emotions not only in adults, but also in children. Aim: The present review aimed at collecting and corroborating the contemporary literature on the prevalence of mental illnesses symptoms and therapeutics interventions relevant to the pandemic of COVID-19. Methods: To compile this review a search of the PubMed electronic database was undertaken using the search keywords such as "novel coronavirus", "COVID-19", "history "prevalence of mental illnesses", "anxiety", "depression" and "post trauma stress" in various transformations and groupings. In addition, search was done about the availability and barriers of psychiatric, psychological and therapeutics interventions related to mental health problems in caused by the pandemics of Covid19. Conclusion: Despite the limited numbers of observational studies in this field to date, it is obvious that, the COVID-19 pandemic has Covid-19 has been a wakeup call to to a forceful and multidimensional response from psychiatrists and allied health professionals, that mental health of people during pandemics should be taken seriously at multiple levels of primary ,secondary and tertiary prevention Therefore, more work needs to be directed toward the development of community health professionals specialists, qualified to address emotional distress and identifying the basic aspect of mental health interventions Further, there is a need to develop mental health interventions which are timelimited, culturally sensitive, and can be taught to healthcare workers and volunteers to be widely disseminated among those working in this field during the pandemics.

Keywords: Pandemics, COVID19, mental illnesses, therapeutics, strategies.

Copyright @ 2020: This is an open-access article distributed under the terms of the Creative Commons Attribution license which permits unrestricted use, distribution, and reproduction in any medium for non-commercial use (NonCommercial, or CC-BY-NC) provided the original author and source are credited.

\section{INTRODUCTION}

Recently, the COVID-19 pandemic has taken the world by a whirlwind. The Severe Acute Respiratory Syndrome Corona Virus 2 (SARS-CoV-2) was recognized as the cause of a disease outbreak that first began in China towards the end of 2019. Since then, the widespread outbreaks of COVID-19, were associated with psychological distress and symptoms of mental illness [1]. The association between COVID-19 and mental illness started due to the inevitable fear and anxiety about the emergence of a new disease. A future so unpredictable can be overwhelming and can cause strong emotions not only in adults, but also in children. In addition, public health actions such as social distancing, drove people to feel more isolated which increased stress and anxiety. It is without doubt however, that these actions were necessary to reduce the rate of transmission of the disease.
With that said, psychiatrists all over the world should be aware of these manifestations, their correlates, and strategies to manage them that encompass both the needs of specific populations [2] and the precautionary measures necessary to contain the spread of COVID-19 [3]. They should also be aware of the gaps present in existing literature, which may need to be filled over time, through research and a more widespread clinical experience.

\section{METHODOLOGY}

The present review is a descriptive review of the contemporary literature on the prevalence of mental illnesses symptoms and interventions relevant to the pandemic of COVID-19. A search of the PubMed electronic database was undertaken using the search keywords such as "novel coronavirus", "COVID-19", "history "prevalence of mental illnesses", "anxiety", "depression" and "post trauma stress" in various 
transformations and groupings. In addition, search was done about the availability and barriers of psychiatric, psychological and therapeutics interventions related to mental health problems in caused by the pandemics of Covid-19.

\section{History of COVID-19}

The first strain of corona virus was discovered in humans in 1930, and then isolated as the Human coronavirus 229E (HCoV-229E) in 1965 [1]. Since then, three other coronaviridae were identified in humans; Human coronavirus NL63 (HCoV-NL63), Human coronavirus OC43 (HCoV-OC43) and Human coronavirus HKU1 (HCoV-HKU1). These were then classified into two groups, alpha and beta. HCoV-229E and HCoV-NL63 are alpha coronavirus $(\alpha-\mathrm{CoV}$ or Alpha-CoV), while HCoV-OC43, HCoV-HKU1, MERS-CoV, SARS-CoV are beta coronavirus $(\beta-\mathrm{CoV}$ or Beta-CoV) [23]. Multiple outbreaks caused by different strains of the virus then began to strike.

In November 2002, an outbreak of Severe Acute Respiratory Syndrome (SARS) was discovered. This disease originated in China and subsequently spread to Vietnam, Hong Kong, Taiwan, Singapore and Canada. In 2012, a new strain with a SARS-like illness, called the Middle East Respiratory Syndrome (MERS) resulted in a limited number of outbreaks, mostly in Saudi Arabia and other middle eastern countries.

Then, in December 2019, the novel coronavirus (nCoV) was identified in Wuhan, China, and was isolated on the $7^{\text {th }}$ of January, 2020 [4]. The World Health Organization then recommended the interim name of the disease as 2019-nCoV Acute Respiratory Disease (2019-nCoV ARD) and 2019 novel corona virus (2019-nCov) as that of the virus [5]. However, the disease was subsequently reclassified as Corona Virus Disease 2019 (COVID-19) and the virus as Severe Acute Respiratory Syndrome Corona Virus-2 (SARS-CoV-2) (7), which is closely related to the earlier SARS-CoV and genetically clustered within the Beta coronavirus with the subgenus Sarbecovirus [6].

In March 2020, the World Health Organization (WHO) finally declared COVID-19 as a pandemic. From then onwards, it was closely monitored and its updates issued by public health groups, such as the U.S. Centers for Disease Control and Prevention (CDC) and the WHO. These groups have also released recommendations for preventing and treating the illness.

\section{Transmission and Symptoms}

Data has shown that the virus spreads from one person to another in close proximity (within about 6 feet, or 2 meters) through respiratory droplets released by the infected person when they happen to cough, sneeze or talk.
In regard to its symptoms, those can vary in severity. While some people may have no symptoms at all, the most common are fever, cough and tiredness. Other reported symptoms may include shortness of breath, muscle aches, chills, sore throat, headache, chest pain, and loss of taste or smell. However, this list is not all inclusive as some rare symptoms have also been registered. Accordingly, symptoms may appear two to 14 days after exposure.

\section{Treatment}

Until today, there is neither a vaccine nor medication available for treating COVID-19. The method of treatment is directed at relieving symptoms by using specific medication, available in pharmacies or hospitals.

\section{Control}

In response to the current coronavirus crisis, most countries and governments began implementing certain control measures to decrease the transmission of the disease. This included the temporary shutdown of unnecessary businesses and schools, prohibiting large gatherings, and calling for quarantines for travelers, as well as encouraging social distancing and wearing masks. A majority of countries have even announced mandatory stay-at-home instructions for all but essential workers.

\section{COVID-19 and the prevalence of mental illness}

Prior to the COVID-19 pandemic, approximately one in five of U.S. adults (47 million) reported having a mental illness in the past year, in addition to more than 11 millions of which were serious. Such reports claimed to cause frequent functional impairments that seriously bound daily activities. Not only that, but the prevalence of depression and anxiety were also high, where in 2017 2018, nearly 17 million adults and an additional 3 million adolescents had a major depressive episode. Moreover, in 2018, nearly a third $(32.5 \%)$ of adults reported feeling worried, nervous, or anxious on a monthly, weekly, or even a daily basis.

Therefore, an extensive body of research has linked social isolation and loneliness to both poor mental and physical health. Moreover, studies regarding the psychological impact of quarantine during other disease outbreaks indicated that such confinement can affect mental health negatively. Therefore, there is a particular concern regarding not only depression, but also suicidal ideation during this time.

\section{Literature related to the association between mental health and COVID-19 in vulnerable populations \\ With many countries implementing social distancing, there have been major changes in national behavior patterns and shutdowns of daily functions. While this may slow the spread and flatten the curve of}


the disease, the consequences on mental health remain unknown, and unacknowledged.

However, it has been evidenced that largescale disasters, whether traumatic, natural or environmental are almost always accompanied by an increase in depression, posttraumatic stress disorder (PTSD), substance use disorder, a broad range of other mental and behavioral disorders, domestic violence, and even child abuse. For example, 5\% of the population affected by Hurricane Ike in 2008, met major depressive disorder criteria about a month later. Similarly, $10 \%$ of New Yorkers showed signs of major depressive disorder in the month following the 9/11 attack, in addition to $25 \%$ who reported increased alcohol use.

When speaking specifically, the Severe Acute Respiratory Syndrome (SARS) pandemic was linked to increases in PTSD, stress, and psychological distress in both patients and clinicians. COVID-19, of course, is closely related to SARS as the virus has been named SARS-CoV-2.

Accordingly, Pappa, Ntella, and Katsaounou [7] reported in a systematic and meta-analysis survey, that the prevalence of anxiety was estimated to be $23.2 \%$ from 12 studies while that of depression was $22.8 \%$ from 10 studies. Moreover, the subgroup analysis revealed that gender and occupational differences caused female HCPs and nurses to exhibit higher rates of affective symptoms compared to male and medical staff respectively. In regards to the prevalence of insomnia, it was estimated to be $38.9 \%$ across 5 studies. This information was collected from thirteen studies with a combined total of 33,062 participants.

Since the virus emerged from China, it only seemed fair to also include the prevalence of such diseases post the pandemic. Therefore, a cross sectional study conducted by Yeen Huang and Ning Zhao [8], reported that the overall prevalence of GAD, depressive symptoms, and sleep quality were $35.1 \%, 20.1 \%$, and $18.2 \%$ respectively. In regards to the occupational group, healthcare workers were also still more likely to have poor sleep quality.

In addition, seven publications (correspondence, $\mathrm{n}=6$; commentary, $\mathrm{n}=1$ ) have identified the vulnerable groups that may be affected by mental illness as a result of the pandemic. This included older adults [2], the homeless [9], migrant workers [10], the mentally ill [11], pregnant women [12] and Chinese students studying overseas [13]. Not only that but another study done by Huang and Ning Zhao [8], found that younger people reported a significantly higher prevalence of GAD and depressive symptoms than those older.
Moreover, two reports from china concerned with practicing psychiatrists [11] regarding COVID-19 and patients with pre-existing psychiatric illness demonstrated that a single outbreak of COVID-19, affected about 50 patients and 30 staff members in a hospital. This may have been due to overcrowding, lack of general medical facilities in psychiatric hospitals, lack of knowledge among mental health professionals, and difficulty in obtaining the cooperation from patients to implement preventive measures, especially those suffering from psychotic disorders [14]. It is as well no surprise that patients with pre-existing mental disorders may be at a higher risk of relapse or newer episodes due to the stress associated with the COVID-19 outbreak [11]. During this period, it is thereby crucial that psychiatrists familiarize themselves with screening and triage procedures, and work closely with physicians and public health specialists to minimize the risks that their patients face [14].

With that being said, other specific issues persist in regards to the other populations listed above. This primarily included the high rates of pre-existing depressive symptoms in the elderly and their lack of access to mental health services [2], the fear of uncontrolled admission or imprisonment among the homeless which may then act as a barrier to mental health care [9], the requirement for outreach and social support among immigrants to reduce the risk of common mental disorders [10], the relationship between COVID-19 - related stress and anxiety and adverse maternal and neonatal outcomes [12], and the inevitable intolerance faced by Chinese students overseas during the pandemic which leads to anxiety and stress-related disorders [13]. In all these cases, it is without doubt that the close collaboration between psychiatrists and specialties from other branches of medicine, as well as with the local authorities and health workers in the community, is essential.

Similarly, in a tracking poll study conducted for American people, it was reported that $57 \%$ of the population have had their lives majorly disrupted by the pandemic, while the remaining 44\% said that it was only minor. Those two groups were thereby more likely to report negative mental health impacts than those who say their lives have only been disrupted "a little" or "not at all" (28\%) [15].

It was also they reported that the refugees $(47 \%)$ reported negative mental health effects resulting from worry or stress related to COVID-19. This was significantly higher than the rate of reports from nonrefugees $(37 \%)$.

Unfortunately, it is therefore believed that after the existing pandemic, there could be a significant increase in anxiety and depression, substance use, loneliness, and domestic violence (specifically child abuse, as there is widespread school closures). 
Recently, a research was conducted with nurses and physicians who were involved in the treatment of COVID-19, and reported a high incidence of stress, anxiety and PTSD, where the levels of anxiety in women and nurses were higher compared to men and physicians, respectively. This can be clarified by the fact that nurses have longer work shifts and closer contact with patients, which can easily lead to fatigue and tension. On the other hand, a study with a similar sample found that the physicians' level of social support was significantly associated with efficacy and quality of sleep, and negatively associated with anxiety and stress [16]. In fact, health care professionals who are in direct contact with infected patients need to have their mental health regularly examined and assessed, especially in relation to depression, anxiety and suicidal ideation. Similarly, it is essential to identify professionals who are vulnerable to psychological exhaustion or with a history of psychosocial risk factors. Therefore, special attention of psychiatric treatments should be provided to those with more serious mental health problems.

More specifically and in the context of COVID-19, it is important to identify secondary psychosocial factors that may potentially affect the mental health of healthcare professionals, and generate stress, e.g., professionals with chronic diseases, living with young children or older family members.

\section{Psychiatric, Psychological and Therapeutic Strategies}

As regard to the psychological and psychiatric interventions, only five studies (correspondence, $\mathrm{n}=2$; commentary, $n=3$ ) were found these articles have directly addressed the use of specific therapeutic approaches to deliver mental health care to persons affected by the COVID-19 epidemic $[17,3,16,18,1]$.

The significance role of psychiatrists during the COVID-19 pandemic was determined on a research from India. This article specifies six important roles for the psychiatrist including: a) public education about the common psychological effects of a pandemic, b) encouraging people to adopt strategies for disease prevention and health promotion, c) participating with the available health care services, d) educating problem-solving techniques to cope with the current crisis, e) empowering patients with COVID-19 and their families and caregivers, and f) providing mental health care services to healthcare workers managing patients with COVID19 [19].

Many therapeutic strategies were suggested for execution for dealing with the emotional helplessness and mental suffering stated by some professionals. One of such strategies was to create proper spaces for listening and exchanging feelings in order to prevent burnout. Besides, psychological support measures should be taken into consideration with the health care professionals' emotional expression. Psychological intervention (PI) during and after crisis aims to teach coping strategies to deal with symptoms of obsession, intrusive thoughts and anticipatory or situational anxiety. Moreover, psychological interventions that aim to facilitate intra-team support, empathy, and compassion towards more vulnerable weak health professionals [16]. Besides, safe healthcare settings, availability and accessibility of economic and human resources, online psychological counseling and psychotherapy and methods to promote mental health are recommended. Xiao et al., [16] stated that, digital communication can also be a viable complementary strategy and should be available 24 hours a day.

In conclusion, the aim of implementing such strategies is to provide mental health services in an easily accessible manner without any increase in the risk of infection. However, they depend significantly on the availability of trained manpower and infrastructure, and it is not known to what extent these advances will be accepted by the general public. Moreover, they have not yet been tested for validation in the respective target populations.

\section{CONCLUSION AND RECOMMENDATIONS}

Despite the limited numbers of observational studies in this field to date, it is obvious that, the COVID-19 pandemic has led to a forceful and multidimensional response from psychiatrists and allied health professionals, that mental health of people during pandemics should be taken seriously at multiple levels of primary, secondary and tertiary prevention. However, the number of patients affected by this pandemic continues to increase, the psychiatric and mental health profession - particularly in Asian and African countries - faces not only the challenge of addressing the numerous barriers and limitations identified but also an opportunity.

Khalil [20], stated that, negative attitude, and stigmatization toward mental illness and people working in psychiatric hospitals might be due to the limitations of health services regarding the presence of awareness programs and insufficient educational resources about mental health and illnesses. Other causes that can explain these disparities could be related to few numbers of psychiatric hospitals at every big city, few numbers of psychiatrists and psychologist and there is no certification programs or special training programs for nurses working in psychiatric hospitals setting. There are greater opportunities for mental health nurses to play a significant role in improving both the physical and mental health of people with serious mental illness along with changing the attitude of public attending to the hospital toward mentally ill patients [20]. There is certainly an increased awareness in most Arab countries of the importance of mental health problems as part of the total health care picture however, this awareness is rarely translated into actions 
on the ground. In addition, most medical and nursing schools in the Arab world have a rudimentary psychiatric program, and it is mostly in the form of scattered lectures and short rotations in clinics or wards [21]. Fortuitously, the increasing number of patients having mental disorders during the pandemics of Covid19 had given the attentions of all governments in every countries to the importance of mental health issues and the mental health care providers to manage the people during and after the pandemics.

\section{RECOMMENDATIONS}

Despite the fact in the above literature, there is an opportunity to implement those suggestions or recommendations which are feasible at a local or regional level as the followings:

- More work needs to be directed toward the development of community health professionals specialists, qualified to address emotional distress and identifying the basic aspect of mental health interventions [17]. Further, there is a need to develop mental health interventions which are time-limited, culturally sensitive, and can be taught to healthcare workers and volunteers. Once developed, such procedures should be widely tested, so that the information regarding effective therapeutic strategies can be circulated among those working in this field.

- Development of proposals to educate the public about the psychobiological underpinnings of psychiatric disorders and about the value of effective treatments. A better understanding of these disorders amongst the public is presumed to decrease stigmatization and encourage the use of currently available and effective strategies.

- Conducting online surveys to assess the scope of mental health problems [3]; the development of online materials for mental health education [3]; the provision of online counselling and self-help services [3] in form of structured letters as a form of asynchronous telepsychiatry consultation [22]; the development of synchronous telemedicine services for diagnostic purposes as well as counselling [18]; and the need to make online mental health services accessible to individuals from lower socioeconomic strata [11].

- There is a need for further research, even in the form of preliminary or pilot studies, to assess the scope of this pandemic in other countries, and the impact of COVID-19 on other vulnerable populations, such as children and adolescents, and those in remote or rural areas who face barriers in accessing health care, and those belonging to lower socioeconomic strata [17].
Acknowledgement: None.

Disclosure of funding sources: Not applicable.

Financial Disclosure: None.

Declaration of Competing Interest: The authors declare that there was no conflict of interest.

\section{REFERENCES}

1. Ward, P. (2020). 'COVID-19/SARS-CoV-2 Pandemic', Faculty of Pharmaceutical Medicine blog, 6 April. Available at: https://www.fpm.org.uk/blog/covid-19-sarscov-2-pandemicl

2. Yang, Y., Li, W., Zhang, Q., Zhang, L., Cheung, T., \& Xiang, Y. T. (2020). Mental health services for older adults in China during the COVID-19 outbreak. Lancet Psychiatry. 7(4):e19.

3. Liu, S., Yang, L., Zhang, C., Xiang Y. T., Liu, Z., Hu, S., \& Zhang, B. (2020). Online mental health services in China during the COVID-19 outbreak. Lancet Psychiatry. 7(4):e17-e18.

4. World Health Organization. (2020). "Novel Coronavirus - China". 12 January 2020. Retrieved 18 May 2020.

5. World Health Organization. (2020). "Novel Coronavirus (2019-nCoV)". 30 January 2020. Retrieved 18 May 2020.

6. European Centre for Disease Prevention and Control. (2020). "Disease background of COVID19". 8 May 2020. Retrieved 18 May 2020.

7. Pappa, S., Ntella, V., Giannakas, T., Giannakoulis, V. G., Papoutsi, E., \& Katsaounou, P. (2020). Prevalence of depression, anxiety, and insomnia among healthcare workers during the COVID-19 pandemic: A systematic review and metaanalysis. Brain, behavior, and immunity.

8. Huang, Y., \& Zhao, N. (2020). Generalized anxiety disorder, depressive symptoms and sleep quality during COVID-19 outbreak in China: a web-based cross-sectional survey. Psychiatry research, 112954.

9. Tsai, J., \& Wilson, M. (2020). COVID-19: a potential public health problem for homeless populations. The Lancet Public Health, 5(4), e186e187.

10. Liem, A., Wang, C., Wariyanti, Y., Latkin, C. A., \& Hall, B. J. (2020). The neglected health of international migrant workers in the COVID-19 epidemic. The Lancet Psychiatry, 7(4), e20.

11. Yao, H., Chen, J. H., \& Xu, Y. F. (2020). Rethinking online mental health services in China during the COVID-19 epidemic. Asian journal of psychiatry, 50, 102015.

12. Fakari, F. R., \& Simbar, M. (2020). Coronavirus Pandemic and Worries during Pregnancy; a Letter to Editor. Archives of Academic Emergency Medicine, 8(1):e21. 
13. Zhai, Y., \& Du, X. (2020). Mental health care for international Chinese students affected by the COVID-19 outbreak. Lancet Psychiatry. 7(4):e22.

14. Zhu, N., Zhang, D., Wang, W., Li, X., Yang, B., Song, J., ... \& Niu, P. (2020). A novel coronavirus from patients with pneumonia in China, 2019. New England Journal of Medicine.

15. Kirzinger, A., Kearney, A., Hamel, L., \& Brodie, M. (2020). KFF health tracking poll-early April 2020: the impact of coronavirus on life in America. 2020.

16. Xiao, H., Zhang, Y., Kong, D., Li, S., \& Yang, N. (2020). The effects of social support on sleep quality of medical staff treating patients with coronavirus disease 2019 (COVID-19) in January and February 2020 in China. Medical science monitor: international medical journal of experimental and clinical research, 26, e9235491.

17. Duan, L., \& Zhu, G. (2020). Psychological interventions for people affected by the COVID19 epidemic. Lancet Psychiatry; 7(4):300-302.
18. Zhou, X., Snoswell, C. L., Harding, L. E., Bambling, M., Edirippulige, S., Bai, X., \& Smith, A. C. (2020). The role of telehealth in reducing the mental health burden from COVID19. Telemedicine and e-Health, 26(4), 377-379.

19. Banerjee, D. (2020). The COVID-19 outbreak: Crucial role the psychiatrists can play. Asian journal of psychiatry, 50, 102014.

20. Khalil, A. I. (2017). Stigma versus mental health literacy: Saudi public knowledge and attitudes towards mental disorders. International Journal for Innovation Education and Research, 5(3), 5977.

21. Yehia, M. (2012). Interview: Dealing with mental illness in the Middle East. 103.

22. Xiao, C. (2020). A novel approach of consultation on 2019 novel coronavirus (COVID-19)-related psychological and mental problems: structured letter therapy. Psychiatry Investig. 17(2):175-176.

23. Centers for Disease Control and Prevention. (2020). "Human Coronavirus Types". 15 February 2020. Retrieved 18 May 2020. 